\title{
Atuação do enfermeiro obstétrico na perspectiva das epistemologias do Sul
}

\author{
Performance of nurse-midwives from the perspective of Epistemologies of the South \\ Desempeño de la enfermera obstétrica en la perspectiva de epistemología del Sur
}

Alexandra do Nascimento Cassiano ${ }^{1}$ (D)

Rejane Maria Paiva de Menezes $^{1}$ (D)

Soraya Maria de Medeiros $^{1}$ (i)

Carlos Jordão de Assis Silva ${ }^{1}$ (1)

Mônica Cristina Ribeiro Alexandre

d'Auria de $\operatorname{Lima}^{1}$ (D)

1. Universidade Federal do Rio Grande do Norte. Natal, RN, Brasil
Autor correspondente:

Alexandra do Nascimento Cassiano

E-mail: ancenfa@hotmail.com.

Recebido em 11/03/2020.

Aprovado em 25/05/2020.

DOI:https://doi.org/10.1590/2177-9465-EAN-2020-0057

\section{RESUMO}

Objetivo: refletir acerca da atuação do enfermeiro obstétrico na atenção à mulher durante o processo parturitivo, sob a perspectiva teórica das Epistemologias do Sul. Método: trata-se de estudo do tipo reflexivo, com base em uma revisão narrativa da literatura. Resultados: após leitura e análise crítica dos documentos selecionados, e com base no referencial teórico das Epistemologias do Sul, emergiram dois eixos temáticos para reflexão, sendo eles: Descolonização do saber: atuação do enfermeiro obstétrico na reorientação do modelo de atenção à mulher durante o processo parturitivo; e O pensamento pós-abissal e a ecologia dos saberes versus práticas humanizadas de atenção ao parto e nascimento. Conclusão e implicações para a prática: o enfermeiro obstétrico contribui para a valorização do conhecimento de grupos sociais poucos expressivos (minoritários), oprimidos ante à hegemonia de um modelo assistencial. Sua postura profissional busca a integração de distintos conhecimentos. A reflexão sobre a assistência da enfermagem obstétrica na perspectiva das Epistemologias do Sul poderá suscitar a discussão sobre as práticas obstétricas vigentes de modo a propulsionar a adoção de cuidados qualificados e humanizados na atenção à mulher durante o parto.

Palavras-chave: Enfermagem Obstétrica; Parto Humanizado; Conhecimento; Prática Profissional; Direitos da Mulher.

\section{ABSTRACT}

Objective: to reflect on the role of certified nurse-midwives in caring for women during parturition from the theoretical perspective of Epistemologies of the South. Method: this is a reflective study based on literature narrative review. Results: after reading and critically analyzing the selected documents, and based on Epistemologies of the South theoretical framework, two thematic axes for reflection emerged, namely: Decolonization of knowledge: performance of nurse-midwives in rearranging a model of care for women during parturition; and Post-abyssal thinking and knowledge ecology vs humanized practices in childbirth and birth care. Conclusion and Implications for practice: nurse-midwives contribute to the valorization of the knowledge of few expressive social groups (minorities), oppressed before the hegemony of a care model. Their professional attitude seeks integration of different knowledge. Reflection on nursing-midwifery care from the perspective of Epistemologies of the South may prompt discussion about current nursing-midwife practices in order to encourage adoption of qualified and humanized care for women during childbirth.

Keywords: Obstetric Nursing; Humanizing Childbirth; Knowledge; Professional Practica; Womens's Rights

\section{RESUMEN}

Objetivo: reflexionar sobre el papel de la enfermera obstétrica en el cuidado de las mujeres durante el proceso de parto, bajo la perspectiva teórica de las Epistemologías del Sur. Método: este es un estudio reflexivo, basado en una revisión literatura narrativa. Resultados: después de leer y analizar críticamente los documentos seleccionados, y basándose en el marco teórico de las Epistemologías del Sur, surgieron dos ejes temáticos para la reflexión, a saber: Descolonización del conocimiento: el papel de la enfermera obstétrica en la reorientación de la modelo de atención a la mujer durante el proceso de parto; y El pensamiento post abisal y la ecología del conocimiento versus prácticas humanizadas en el parto y el cuidado del parto. Conclusión e implicaciones para la práctica: la enfermera obstétrica contribuye a la valorización del conocimiento de pocos grupos sociales expresivos (minorías), oprimidos antes de la hegemonía de un modelo de atención. Su postura profesional busca la integración de diferentes conocimientos. La reflexión sobre la atención obstétrica de enfermería desde la perspectiva de las Epistemologías del Sur puede impulsar la discusión sobre las prácticas obstétricas actuales para alentar la adopción de atención calificada y humanizada en el cuidado de las mujeres durante el parto.

Palabras clave: Enfermería Obstétrica; Parto Humanizado; Conocimiento; Prática profissional; Derechos de la Mujer. 


\section{INTRODUÇÃO}

A inserção do enfermeiro obstétrico (EO) no Brasil é facilmente identificável nas políticas públicas de atenção ao parto e nascimento instituídas por volta dos anos 90, em um contexto de crítica e reflexão ao modelo biomédico vigente da obstetrícia, sendo este caracterizado por práticas intervencionistas, relações verticalizadas e perda da autonomia da mulher em seu processo de parturição. ${ }^{1}$ Desde a década dos anos de 1985, a Organização Mundial da Saúde (OMS) já havia publicado o documento "Tecnologia Apropriada para Partos e Nascimentos", o qual seria o marco da transformação do modelo de assistência ao parto no mundo com vistas à humanização da atenção ao parto e nascimento. ${ }^{2}$

$\mathrm{Na}$ realidade brasileira, dentre as medidas adotadas há alguns anos pelo Ministério da Saúde (MS) para a reorientação do modelo assistencial, citam-se as Portarias GM/MS de $n^{\circ}$.2.815/98 e 169/98, que regulamentaram a assistência obstétrica realizada por enfermeiros, incluindo o parto normal realizado pelo profissional na lista de procedimentos do Sistema Único de Saúde (SUS) e a emissão de Autorização de Internação Hospitalar (AlH) assinada pelo mesmo. Nesse sentido, destacam-se a Lei n 7.498 de 1986 e o Decreto $n^{\circ} 94.406$ de 1987, que regulamentam o exercício profissional da enfermagem, com diretrizes e normas para a atuação do EO na assistência ao parto normal, sendo esse responsável por assistir à parturiente, identificar distócias, realizar epsiotomia, epsiorrafia e anestesia local quando necessário.

Posteriormente, em 2000, o Programa de Humanização do Pré-natal e Nascimento (PHPN) consolidou a participação do profissional enfermeiro como co-participante da Política Nacional de Atenção ao Parto (PNAP), objetivando incentivo ao parto normal, além da redução dos indicadores de morbidade e mortalidade materna e neonatal, das taxas de cesarianas e de intervenções desnecessárias durante o trabalho de parto e parto. ${ }^{3}$

Na perspectiva de um modelo de assistência humanizada, a atuação do EO centra-se na fisiologia do parto, respeitando as necessidades e o protagonismo feminino, em detrimento da objetificação do corpo da mulher às intervenções biomédicas. Além disso, em um parto dito humanizado, faz-se amplo uso de medidas não farmacológicas para alívio da dor, como também de evidências científicas sem se abrir mão de outros saberes populares e/ou culturais, que propiciam satisfação, qualidade e participação da mulher e seus familiares durante o processo de parturição.

Esses cuidados, sob o ponto de vista teórico da Epistemologias do Sul ${ }^{4}$, contrapõem-se à hegemonia do conhecimento biomédico já estabelecido e reconhecido como a Epistemologia do Norte. Essa nova perspectiva tem o intuito de resgatar a autonomia da mulher, permitir a presença de outras pessoas (familiares e doulas) no momento do parto, além de valorizar um diálogo entre os conhecimentos, saberes e experiências até então pouco valorizados.

Ante o exposto, o presente artigo tem por objetivo refletir acerca da atuação do enfermeiro obstétrico na atenção à mulher durante o processo parturitivo, sob a perspectiva teórica das
Epistemologias do Sul. Portanto, este estudo teórico do tipo reflexivo pretende responder a seguinte questão norteadora: de que forma a perspectiva teórica das Epistemologias do Sul perpassa e aprimora a atuação do enfermeiro obstétrico na atenção à mulher que vivencia o processo parturitivo?

Para tanto, será realizada uma revisão narrativa da literatura por meio de busca em livros, artigos e documentos que versam sobre a temática estudada, a fim de discuti-la sob um ponto de vista teórico/contextual. ${ }^{5}$ Com esse propósito, realizou-se a leitura do livro "Epistemologia do Sul" e de artigos científicos ${ }^{1,3,6}$ que intermediassem a articulação entre a enfermagem obstétrica e o referencial teórico das Epistemologias do Sul. Também, foram referenciados documentos e manuscritos que abordam a atuação do EO no contexto da atenção ao parto e nascimento sob uma perspectiva política e humanizada.

A busca dos artigos científicos em periódicos eletrônicos foi realizada no Scientific Electronic Library Online (SciELO Brasil) e no Banco de Dados de Enfermagem (BDENF), em junho de 2019, utilizando os seguintes descritores: enfermagem obstétrica e parto humanizado, com o uso do operador boleando "AND" entre eles. Foi possível identificar 268 documentos científicos, dos quais 16 eram artigos científicos que abordavam sobre a atuação do EO e humanização da atenção ao parto e nascimento, compondo, assim, material para este estudo reflexivo.

Por conseguinte, emergiram dois eixos de reflexão, os quais foram: Descolonização do saber: atuação do enfermeiro obstétrico na reorientação do modelo de atenção à mulher durante o processo parturitivo; e O pensamento pós-abissal e a ecologia dos saberes versus práticas humanizadas de atenção ao parto e nascimento.

\section{REFERENCIAL TEÓRICO}

Boaventura de Sousa Santos ${ }^{4}$ é considerado um dos sociólogos mais influentes da sociedade contemporânea. Seus trabalhos possuem importante relevância teórica para as diferentes disciplinas das ciências sociais e humanas brasileiras, como direito, educação, serviço social, ciência política e sociologia, assim como para as ciências da saúde e, em especial para a enfermagem.

Das suas obras publicadas, destaca-se a teoria da "Epistemologias do Sul" como uma concepção teórica que busca compreender o contexto sócio-histórico mundial e fazer um contraponto com o modelo hegemônico da produção e reprodução social do conhecimento ditado pelo capitalismo.

Etimologicamente, a origem do termo "epistemologia" significa discurso ou estudo sobre a ciência. Essa compreende o estudo crítico dos princípios, hipóteses e resultados das diversas ciências, ou seja, trata-se da teoria do conhecimento. ${ }^{7}$ Para Boaventura e Meneses ${ }^{4}$, a epistemologia é toda noção ou ideia, refletida ou não, sobre as condições do que conta como conhecimento válido.

Logo, Epistemologias do Sul representam a diversidade epistemológica do mundo, que compreende a diversidade de conhecimentos, onde o Sul é concebido metaforicamente como 
um campo de desafios que busca superar os danos causados pela relação colonial do capitalismo europeu sob os países e regiões do Sul geográfico. ${ }^{4}$

Dentre os elementos conceituais que constituem o escopo do referencial Epistemologias do Sul, citam-se a colonização do saber e a linha abissal, as quais, à luz do pensamento de Boaventura, desdobram-se nos constructos da descolonização do saber, pensamento pós-abissal e ecologia de saberes.

$\mathrm{Na}$ tentativa de compreender sobre a descolonização do saber, entende-se que ela consiste na recuperação dos saberes de grupos sociais que, em decorrência do modelo colonialista do capitalismo, foram historicamente e socialmente colocados como objetos dos saberes dominantes do Norte, concretizados na ciência moderna e considerados durante séculos como os únicos válidos e legítimos. ${ }^{4,6}$

Em consonância com esse entendimento, compreende-se o pensamento pós-abissal como parte do pressuposto de que a diversidade epistemológica do mundo permanece por construir-se, pois a diversidade de conhecimentos é inesgotável. Por conseguinte, a ecologia dos saberes é o interconhecimento resultante do reconhecimento da pluralidade de conhecimentos heterogêneos, sendo a ciência moderna apenas um deles, em interação sustentável e dinâmica entre todos, de modo a não comprometer a autonomia de cada um deles. ${ }^{4}$

Nesse âmbito teórico conceitual sobre as Epistemologias do Sul, tem-se como propósito fazer uma reflexão teórica com relação à atuação do EO na atenção humanizada à mulher no período do parto, cuja compreensão se respalda na abordagem de Boaventura e Meneses ${ }^{4}$. Portanto, será possível subsidiar o processo de reflexão acerca do campo de conhecimento e práticas da enfermagem obstétrica, com base no reconhecimento da pluralidade de conhecimentos, suas interações e autonomia.

Assim, os tópicos a seguir têm a pretensão de fazer uma interlocução entre os conceitos definidos anteriormente e a atuação do EO como precursor da humanização na atenção ao parto e nascimento.

\section{RESULTADOS E DISCUSSÃO}

\section{Descolonização do saber: atuação do enfermeiro obstétrico na reorientação do modelo de atenção à mulher durante o processo parturitivo}

Compreender o significado de "descolonização do saber" exige de qualquer profissional o entendimento prévio do que seja a sua "colonização". Ao se pensar ou refletir sobre o conhecimento, há uma zona colonial correspondente ao universo de crenças, comportamentos e saberes que são desconsiderados como "conhecimento", por não estarem circunscritos no julgamento ocidental de verdadeiro e falso. Tal pensamento baseia-se na ciência moderna européia, que divide o mundo em humano e sub-humano, mediante a força da linha abissal, e determina a segregação constante entre o conhecimento válido cientificamente e os não válidos. Portanto, a colonização do saber significa, de modo denotativo, um modelo de exclusão e exploração radical, assim como outrora acontecia no modelo explorador colonial. ${ }^{4}$

A prática assistencial da obstetrícia foi exercida durante séculos por mulheres denominadas de parteiras ou aparadeiras, que, durante o século $X X$, na vigência do desenvolvimento tecnológico da assistência médica, foram denominadas de profissionais com pouco conhecimento científico, designadas ao subúrbio da assistência ao parto. Nesse contexto, os conhecimentos empíricos, herdados de geração a geração, e que se constituíam em um corpo de conhecimento intuitivo do fazer da parteira, foram colocados para além da linha abissal, ou seja, foram colonizados em detrimento do saber ocidental da medicina moderna. A partir disso, a construção histórica e social dessa profissão se pauta em relações de poder, inclusive de gênero, sob o manto da ciência verdadeira.

Como resultado, introduziu-se de forma rotineira, práticas medicalizadas no processo parturitivo, tornando o nascimento um evento hospitalar centrado na figura do médico e não na da mulher. Nessa perspectiva, esta, tem retirada a sua autonomia e é submetida a procedimentos desnecessários como restrição de dieta, enema, tricotomia, amniotomia precoce, posição litotômica, epsiotomia, manobra de kristeller, parto cirúrgico (fórceps ou cesárea) sem critérios de indicação, entre outros. ${ }^{8}$

Apesar de as inovações tecnológicas terem contribuições inegáveis como a redução da mortalidade materna e neonatal, como por exemplo pela indicação do parto cesariano diante das urgências obstétricas e fetais, o uso inadequado de tecnologias e de intervenções desnecessárias sem evidências científicas durante o parto, consubstancia-se enquanto condutas iatrogênicas que podem trazer prejuízos à saúde do binômio mãe e filho. ${ }^{9}$ Logo, o que se questiona é a utilização rotineira desses procedimentos e, principalmente, a exploração/colonização do corpo feminino como objeto de intervenção, sem que Ihes seja permitido à vivência do parto conforme sua necessidade ou possibilitado condições de acesso a outras formas de conhecimento e saberes que propiciem a qualificação e humanização do cuidado.

Diante desse contexto e na perspectiva da Epistemologias do Sul, a transformação do modelo de atenção ao parto humanizado perpassa pela descolonização do saber, pois essa consiste em um conjunto de redes, iniciativas, organizações e movimentos que têm como princípios a igualdade e o reconhecimento das diferenças na luta contra a exclusão, seja ela econômica, social, política, cultural, ou nas relações estabelecidas durante o cuidado. ${ }^{4}$ Por defender que a compreensão de mundo excede a compreensão científica ocidental, representada na obstetrícia pela hegemonia médica no cenário do parto, a descolonização advoga em favor da hibridização desse conhecimento, a exemplo da atuação do EO na assistência ao parto.

Resultados de estudos indicam que nos países em que a atenção ao parto segue o modelo centrado nos preceitos da fisiologia e da dignidade da mulher, associados à valorização dos EO, apresentam redução dos indicadores de morbidade e mortalidade materna e neonatal, assim como menores índices de intervenções desnecessárias e taxa de cesariana. ${ }^{10}$ 
Isso ocorre devido à atuação do EO, cuja responsabilidade inclui, além dos princípios de humanização, como respeito, empatia, intersubjetividade e formação de vínculo à mulher e ao recém-nascido, uma atenção livre de possíveis iatrogenias. ${ }^{8}$

Um cuidado baseado em evidências científicas que sustentam a ciência da enfermagem, mas que, sobremaneira, se faz pautado na individualidade e singularidade feminina, valoriza o protagonismo da mulher e permite a interlocução da assistência ante a crenças e valores culturais da parturiente. ${ }^{8}$

Nesse sentido, esse profissional tem a competência de acompanhar o processo fisiológico do nascimento, ao passo que é capaz de reconhecer distorcias que necessitem de intervenções. As condutas que possibilitam além dos benefícios diretos às gestantes, recém-nascidos e familiares são o incentivo ao trabalho em equipe, a elaboração de protocolos que contemplem a complementariedade das profissões e adoção de práticas recomendadas, como, por exemplo, as medidas não farmacológicas para alívio da dor. ${ }^{10}$

Esse profissional, qualificado para atuar na assistência ao parto, tem formação realizada pelos cursos de pós-graduação lato sensu, como especializações e programas multiprofissionais de residência em saúde materno infantil e/ou saúde da mulher e de enfermagem obstétrica. Diferentemente do que ocorria no século XIX, no que se refere à formação das parteiras e enfermeiras obstetras em geral, vinculadas às escolas médicas, hoje, os cursos de especialização em enfermagem obstétrica seguem um modelo de ensino com ênfase na compreensão do fenômeno reprodutivo como natural, saudável e centrado na mulher. Também há valorização do conhecimento e saber popular, articulado com o conhecimento científico e demais dimensões da competência profissional, como a habilidade técnica, tomada de decisão, autonomia e valorização do trabalho em equipe interdisciplinar. ${ }^{11}$

Assim sendo, a atuação do EO no cenário do parto constitui-se em uma possibilidade de mudança paradigmática, ou seja, uma forma de expressão do conhecimento da Epistemologia do Sul na reorientação do modelo de atenção à mulher durante o processo parturitivo. A prática desse profissional contribui para a valorização dos conhecimentos de grupos sociais outrora excluídos pelo modelo biomédico e para o resgate do protagonismo e autonomia da mulher durante o trabalho parto e do nascimento de seu filho.

\section{O pensamento pós-abissal e a ecologia dos saberes versus práticas humanizadas de atenção ao parto e nascimento}

O pensamento pós-abissal e a ecologia dos saberes são conceitos que se complementam tanto em relação à sua definição quanto à fundamentação teórico-filosófica. Ambos são produtos oriundos da desconstrução do pensamento abissal, compreendido como a concretização do pensamento moderno ocidental, que não admite a co-presença de dois lados da linha abissal. Em outras palavras, consiste na concessão à ciência moderna do monopólio da divisão entre o que é científico e não científico, entre o que é verdadeiro ou falso, de acordo com seus padrões de demarcação. ${ }^{4}$

Pensar na presença de uma linha abissal inserida nas práticas de atenção humanizadas ao parto e nascimento remete à discriminação entre as condutas consideradas "legitimamente" científicas ou de cunho biologicista, geralmente executadas pela medicina obstétrica, com aquelas estimadas como "humanizadas", comumente realizadas pelos demais profissionais, dentre eles o EO. Soma-se a isso o pensar dividido entre as profissões que limita a troca de saberes e atribui julgamentos de relevância entre os diversos conhecimentos.

Outrossim, é necessária uma reflexão, pois deduzir que médicos não podem prestar assistência ao parto de forma humanizada, ou que realizem o parto aplicando medidas não farmacológicas para alívio da dor, é impedir que o saber contra hegemônico possa ser alcançado de forma democrática. Sob essa perspectiva, questiona-se: os EO possuem conhecimento e habilidades técnicas e científicas compatíveis para realizarem uma avaliação clínica e evolução do trabalho de parto tanto quanto os médicos? Pode-se julgar qual prática é mais importante do que a outra no contexto da assistência ao parto e nascimento? E, por fim, esses saberes não seriam, na verdade, complementares?

Manter-se vinculado à natureza desse pensamento dicotômico significa contribuir com a autorreprodução do pensamento abissal e estimular a disputa entre médicos obstetras e EO pelo direito de exercerem a obstetrícia. ${ }^{4}$

Faz-se necessário, desse modo, um enfrentamento ante à questão que se apresenta, a fim de que o ciclo hegemônico discriminatório não se continue. $O$ pensamento pós-abissal vem a ser um caminho possível para transformação dessa realidade, pois promove a justiça cógnita global. Trata-se de um pensamento alternativo com alternativas, cuja premissa basilar fundamenta-se na condição de que a co-presença de práticas e agentes de ambos os lados da linha é primordial para a construção de uma sociedade contemporânea igualitária. ${ }^{4}$ Corresponde a uma afirmação e o reconhecimento positivo da pluralidade de conhecimentos existentes no mundo, não os limitando àqueles julgados como científicos. ${ }^{6}$

Isso implica a adoção de uma postura profissional que busca integrar os saberes biomédicos, científicos, sociais, econômicos, culturais, populares e das diferentes profissões, a fim de convergi-los em prol da humanização e da qualificação da assistência às mulheres durante o processo parturitivo. Refletir sob essa perspectiva igualmente nos remete à ecologia dos saberes.

A ecologia dos saberes tem como premissa a ideia da diversidade epistemológica do mundo, ou seja, faz renúncia a qualquer epistemologia geral, sendo, por conseguinte, uma contra epistemologia. Segue a utopia de que é possível aprender outros conhecimentos, sem perder de vista o seu próprio, concretizando-se como uma tecnologia da prudência. ${ }^{4}$ É definição de interesse para ciência da enfermagem, posto que a pluralidade de conhecimento está diretamente ligada à clarificação do seu corpo de saberes e práticas, concretizados no conjunto de elementos que compõem seu metaparadigma: pessoa, ambiente, saúde e enfermagem. ${ }^{12}$ 
A compreensão sobre a existência dos saberes heterogêneos nos dá uma amplitude maior de formas de colaboração junto aos sujeitos. Considerar as necessidades das mulheres durante a vivência do parto e do nascimento é uma das preocupações constantes do $\mathrm{EO}$, o que exige do profissional o pensamento ecológico de buscar distintos conhecimentos que comtemplem tais necessidades em diferentes contextos e realidades. Essa postura é condizente com a atenção ao parto e nascimento humanizados.

Destarte, é preciso reconhecer que a humanização diz respeito a um projeto global, reproduzido por diversos atores sociais, mas que precisa ter como norte a opinião da mulher, situando-a como protagonista da cena. Também implica a conduta profissional de respeito à fisiologia do parto, sem intervir, desnecessariamente, e absorver os aspectos sociais e culturais; oferecer o necessário suporte emocional à mulher e sua família; garantir a presença de um acompanhante e respeito aos direitos da mulher. ${ }^{10}$

Sobremaneira, as mulheres devem ser informadas dos procedimentos aos quais serão submetidas, e os profissionais, por sua vez, devem atuar com competência e eficácia enquanto colaboradores da experiência, colocando-se à disposição com o seu conhecimento para manutenção do equilíbrio físico e psíquico da mulher. ${ }^{10}$

Nesse ponto, ressalta-se a atuação do EO no empoderamento da mulher durante o parto e na implementação de práticas integrativas, como as de medidas não farmacológicas para alívio da dor, a exemplo da livre deambulação e escolhas de posição, aromaterapia, cromoterapia, musicoterapia, presença de acompanhante ou de doula, técnicas de respiração, banho de chuveiro morno, massagem e utilização de recursos tecnológicos não invasivos (bola de bobath, escada de sling e rebozo).

Tecnologias utilizadas com base em evidências científicas e seus benefícios para a fisiologia do trabalho do parto e, principalmente, para a satisfação da mulher, são comprovados em revisões sistemáticas da literatura ${ }^{13}$, ao passo que essas são recomendadas pela OMS como uma das estratégias prioritárias para promoção da adequada atenção ao parto e nascimento. ${ }^{14}$

Por isso, considera-se que o EO tem perfil e competência para acompanhar o processo fisiológico do nascimento, contribuindo com a evolução natural do mesmo. Sua atuação respalda-se nos princípios do respeito ao ser humano, na empatia, na intersubjetividade e no vínculo, oferecendo à mulher um cuidado qualificado e humanizado. ${ }^{10}$

\section{CONCLUSÕES E IMPLICAÇÕES PARA A PRÁTICA}

Ao refletir acerca da atuação do EO, com base a perspectiva das Epistemologias do Sul de Boaventura Sousa Santos, foi possível identificar pontos de convergência entre tal referencial e as práticas realizadas por esse profissional no contexto do parto e nascimento.

Em sua atuação, o mesmo contribui para descolonização do saber ao valorizar os conhecimentos de grupos sociais outrora excluídos pelo modelo biomédico, resgatando, inclusive, o protagonismo da mulher na vivência do seu trabalho parto e parto. O pensamento pós-abissal e a ecologia dos saberes se fazem presentes na postura profissional de integrar os conhecimentos biomédicos, científicos, sociais, econômicos, culturais, populares e das diferentes profissões, a fim de convergi-los em prol da humanização e da qualificação da assistência às mulheres durante o parto e nascimento.

A enfermagem obstétrica, portanto, desponta como uma das diferentes formas de expressão das Epistemologias do Sul, posto sua emergência enquanto ciência contrária à visão limitada do biologicismo, de modo a reverberar positivamente sob aqueles que devem ser o centro do cuidado durante o processo parturitivo: a mulher e o seu filho.

Como implicações para o cuidado, destaca-se que refletir sobre assistência da enfermagem obstétrica na perspectiva das Epistemologias do Sul poderá suscitar a discussão sobre as práticas obstétricas vigentes e, a partir disso, propulsionar a adoção de cuidados qualificados e humanizados na atenção à mulher durante o parto.

\section{CONTRIBUIÇÕES DOS AUTORES}

Desenho do estudo, aquisição, análise de dados e interpretação dos resultados, redação e revisão crítica do manuscrito, aprovação da versão final do artigo, responsabilidade por todos os aspectos do conteúdo e a integridade do artigo publicado: Alexandra do Nascimento Cassiano.

Desenho do estudo, aquisição, análise de dados e interpretação dos resultados, redação e revisão crítica do manuscrito, aprovação da versão final do artigo, responsabilidade por todos os aspectos do conteúdo e a integridade do artigo publicado: Rejane Maria Paiva de Menezes e Soraya Maria de Medeiros.

Análise de dados e interpretação dos resultados, redação e revisão crítica do manuscrito, aprovação da versão final do artigo, responsabilidade por todos os aspectos do conteúdo e a integridade do artigo publicado: Carlos Jordão de Assis Silva, Mônica Cristina Ribeiro e Alexandre d'Auria de Lima.

\section{EDITOR ASSOCIADO}

\author{
Ana Luiza de Oliveira Carvalho
}

\section{REFERENCIAS}

1. Camacho KG, Progianti JM. The transformation of nurses' obstetrical practice in humanized birth care. Rev Eletr Enf. 2013;15(3):648-55 http://dx.doi.org/10.5216/ree.v15i3.18588.

2. World Health Organization. Appropriate technology for birth. Lancet 1985;2(8452):436-7. PMid:2863457.

3. Amaral RCS, Alves VH, Pereira AV, Rodrigues DP, Silva LA, Marchior GRS. The insertion of the nurse midwife in delivery and birth: obstacles in a teaching hospital in the Rio de Janeiro state. Esc Anna Nery. 2019;23(1):1-9. http://dx.doi.org/10.1590/2177-9465-ean-2018-0218.

4. Boaventura SS, Meneses MP. Epistemologias do Sul. Coimbra: Editora Cortez; 2017.638 p

5. Rother ET. Systematic literatura review $x$ narrative review. Acta Paul Enferm. 2007;20(2):1-2. http://dx.doi.org/10.1590/S0103-21002007000200001. 


\section{Enfermagem obstétrica e epistemologias do sul}

Cassiano AN, Menezes RMP, Medeiros SM, Assis Silva CJ, Lima MCRAD

6. Rodrigues CCFM, Carvalho DPSRP, Salvador PTCO, Medeiros SM, Menezes RMP, Ferreira Jr MA et al. Innovative nursing education from the perspective of epistemologies of the South. Esc Anna Nery. 2016;20(2):384-9. http://dx.doi.org/10.5935/1414-8145.20160053.

7. Jensen JS, Cruz ER. Epistemologia. REVER [Internet]. 2013. [citado 2019 jul 20];13(2):171-91. Disponível em: https://revistas.pucsp.br/ index.php/rever/article/viewFile/18418/13662

8. Possati AB, Prates LA, Cremonese L, Scarton J, Alves CN, Ressel LB. Humanization of childbirth: meanings and perceptions of nurses. Esc Anna Nery.2017;21(4):1-6. http://dx.doi.org/10.1590/2177-9465-ean-2016-0366.

9. Leal MC, Bittencourt SA, Esteves-Pereira AP, Ayres BVDS, Silva LBRAA, Thomaz EBAF et al. Avanços na assistência ao parto no Brasil: resultados preliminares de dois estudos avaliativos. Cad Saude Publica. 2019;35(7):e00223018. http://dx.doi.org/10.1590/0102-311x00223018. PMid:31340337.
10. Ministério da Saúde (BR). Humanização do parto e do nascimento. Vol. 4. Brasília: Ministério da Saúde/Universidade Estadual do Ceará; 2014. 465 p. Cadernos HumanizaSUS.

11. Riesco MLG, Tsunechiro MA. Formação profissional de obstetrizes e enfermeiras obstétricas: velhos problemas ou novas possibilidades? Rev Estud Fem. 2002;10(2):449-59. http://dx.doi.org/10.1590/S0104026X2002000200014.

12. Queirós PJP. Nursing, an ecology of knowledges. Cultura de los Cuidados. 2016;20(4):137-46. http://dx.doi.org/10.14198/cuid.2016.45.15.

13. Jones L, Othman M, Dowswell T, Alfirevic Z, Gates S, Newburn M et al. Pain management for women in labour: an overview of systematic reviews. Cochrane Database Syst Rev. 2012;3:CD009234. PMid:22419342.

14. World Health Organization. WHO recommendations: intrapartum care for a positive childbirth experience. Geneva: WHO; 2018. 